JOURNAL OF

FUNCTION SPACES AND APPLICATIONS

Volume 2, Number 3 (2004), 267-277
(C) 2004, Scientific Horizon http://www.jfsa.net

\title{
A sharpness result for powers of Besov functions
}

\author{
Gérard Bourdaud
}

(Communicated by Jürgen Appell)

2000 Mathematics Subject Classification. 46E35, 47H30.

Keywords and phrases. Besov spaces, composition operators.

Abstract. A recent result of Kateb asserts that $f \in B_{p, q}^{s}\left(\mathbb{R}^{n}\right)$ implies $|f|^{\mu} \in$ $B_{p, q}^{s}\left(\mathbb{R}^{n}\right)$ as soon as the following three conditions hold : (1) $0<s<\mu+(1 / p)$, (2) $f$ is bounded, (3) $\mu>1$. By means of counterexamples, we prove that those conditions are optimal.

\section{Introduction and main results}

According to Kateb [4], the implication

$$
f \in B_{p, q}^{s}\left(\mathbb{R}^{n}\right) \quad \Longrightarrow \quad|f|^{\mu} \in B_{p, q}^{s}\left(\mathbb{R}^{n}\right)
$$

holds as soon as the following three conditions are satisfied :

(1) $0<s<\mu+(1 / p)$,

(2) $f \in L^{\infty}\left(\mathbb{R}^{n}\right)$,

(3) $\mu>1$.

We denote here by $B_{p, q}^{s}\left(\mathbb{R}^{n}\right)$, the Besov space — see for instance [9] for the definition. Throughout the paper, we assume that $p, q \in[1,+\infty]$ and $s, \mu>0$. In the following three theorems, we shall see that the above conditions are the best possible. 
Theorem 1. Assume that $\mu$ is not an even integer. Then there exists $f \in \mathcal{D}\left(\mathbb{R}^{n}\right)$ such that $|f|^{\mu} \notin B_{p, q}^{\mu+(1 / p)}\left(\mathbb{R}^{n}\right)$, for every $q<\infty$.

Remark. If $\mu$ is an even integer, then $t \mapsto|t|^{\mu}$ is a $C^{\infty}$ function. Such functions are known to act on $B_{p, q}^{s}\left(\mathbb{R}^{n}\right) \cap L^{\infty}\left(\mathbb{R}^{n}\right)$ (cf. [6, Theorem 5.3.6(1), p. 336]).

Theorem 2. Let $\mu>1$. Assume that $s<n / p$ or that $s=n / p$ and $q>1$. Then there exists a positive unbounded function $f$ such that $f \in B_{p, q}^{s}\left(\mathbb{R}^{n}\right)$ but $f^{\mu} \notin B_{p, q}^{s}\left(\mathbb{R}^{n}\right)$.

Now we turn to the sharpness of the condition $\mu>1$. If $\mu<1$, the function $t \mapsto|t|^{\mu}$ is not locally Lipschitz continuous; then by a general result of Bourdaud [1], there exist functions $f$ in $B_{p, q}^{s}\left(\mathbb{R}^{n}\right)$ such that $|f|^{\mu} \notin B_{p, q}^{s}\left(\mathbb{R}^{n}\right)$. Here we want to go further : the same phenomenon can appear even if the function $f$ is bounded and positive.

Theorem 3. If $\mu<1$, there exists a positive function $f \in B_{p, q}^{s}\left(\mathbb{R}^{n}\right) \cap$ $L^{\infty}\left(\mathbb{R}^{n}\right)$ such that $f^{\mu} \notin B_{p, q}^{s}\left(\mathbb{R}^{n}\right)$.

In Section 2, we construct some specific test functions in Besov spaces while in Section 3, we give the proofs of the above three theorems. We denote by $c, c_{1}, c_{2}, \ldots$ various strictly positive constants.

\section{Some functions in Besov spaces}

Let $\rho$ be a $C^{\infty}$ function on $\mathbb{R}$ such that $\rho(x)=1$ for $x \leq e^{-3}$ and $\rho(x)=0$ for $x \geq e^{-2}$. We denote by $\Delta$ the Laplace operator on $\mathbb{R}^{n}$.

Proposition 1. Let $(\alpha, \sigma) \in \mathbb{R}^{2}$. Then the Fourier transform of the function $f$ defined by

$$
f(x):=|\log | x||^{\alpha}(\log |\log | x||)^{-\sigma} \rho(|x|),
$$

is indefinitely differentiable on $\mathbb{R}^{n}$ and satisfies

$$
\Delta^{k}(\widehat{f})(\xi)=O\left(|\xi|^{-n-2 k}(\log |\xi|)^{\alpha-1}(\log (\log |\xi|))^{-\sigma}\right),
$$

as $|\xi| \rightarrow \infty$, for any $k \in \mathbb{N}$. In case $\alpha=0$, the above estimation can be improved as follows :

$$
\Delta^{k}(\widehat{f})(\xi)=O\left(|\xi|^{-n-2 k}(\log |\xi|)^{-1}(\log (\log |\xi|))^{-\sigma-1}\right) .
$$

Proof. This is probably a known result. We outline the proof, following the method of S. Wainger [10, Theorem 3, p. 27]. We need a few notations : 
- $J_{\mu}$ is the classical Bessel function (see e.g. [11]) ;

- $h_{0}(t):=|\log t|^{\alpha}(\log |\log t|)^{-\sigma}$, for $0<t<1 / e$;

- $u_{k}(r):=\int_{0}^{\infty} J_{\frac{n}{2}-1}(r t) h_{0}(t) t^{\frac{n}{2}+2 k} \rho(t) d t$

- $h_{m}(t):=t h_{m-1}^{\prime}(t)(m=1,2, \ldots)$.

An easy computation yields the formula

(2) $h_{m}(t)=(-1)^{m}|\log t|^{\alpha-m}(\log |\log t|)^{-\sigma}\left(\sum_{j=0}^{m} b_{\alpha, \sigma, j, m}(\log |\log t|)^{-j}\right)$,

with $b_{\alpha, \sigma, 0, m}=\alpha(\alpha-1) \cdots(\alpha-m+1)$. Then we have

$$
\Delta^{k}(\widehat{f})(\xi)=(-1)^{k}(2 \pi)^{n / 2}|\xi|^{1-\frac{n}{2}} u_{k}(|\xi|) \quad, \quad \forall \xi \neq 0 .
$$

First of all, we establish the following alternative formula for $u_{k}$ :

$$
u_{k}(r)=r^{-\nu} \int_{0}^{\infty} t^{\frac{n}{2}+2 k} J_{\frac{n}{2}+\nu-1}(r t)\left(\sum a_{\nu, m, j, l} t^{-m-l} h_{m}(t) \rho^{(j)}(t)\right) d t
$$

for any integer $\nu \geq k+1$, where the summation is extended to all the triples $(m, j, l) \in \mathbb{N}^{3}$ such that $m+j+l=\nu$ and such that

$$
m=0 \quad \Longrightarrow \quad j \geq 1
$$

Proof of (3). It is essentially the Lemma 8 of Wainger [10]. We first prove (3), for any $\nu \geq 1$ and without the restriction (4), by using repeated integrations by parts and the identity

$$
J_{\nu}(t r)=\frac{1}{r} t^{-\nu-1} \frac{d}{d t}\left(t^{\nu+1} J_{\nu+1}(t r)\right)
$$

(see $[11$, p. 45]). If we take $\nu=k$, the formula becomes

$$
u_{k}(r)=r^{-k} \int_{0}^{\infty} t^{\frac{n}{2}+2 k} J_{\frac{n}{2}+k-1}(r t)\left(\sum a_{k, m, j, l} t^{-m-l} h_{m}(t) \rho^{(j)}(t)\right) d t .
$$

In the above formula, we consider the term corresponding to $m=j=0$, i.e. $l=k$, and we perform a further integration by parts; by (5), we obtain 


$$
\begin{aligned}
\int_{0}^{\infty} & t^{\frac{n}{2}+k} J_{\frac{n}{2}+k-1}(r t) \rho(t) h_{0}(t) d t \\
= & \frac{1}{r} \int_{0}^{\infty} \frac{d}{d t}\left(t^{\frac{n}{2}+k} J_{\frac{n}{2}+k}(r t)\right) \rho(t) h_{0}(t) d t \\
& =-\frac{1}{r} \int_{0}^{\infty} t^{\frac{n}{2}+k} J_{\frac{n}{2}+k}(r t)\left(\rho^{\prime}(t) h_{0}(t)+\rho(t) h_{0}^{\prime}(t)\right) d t .
\end{aligned}
$$

By this way, we obtain the desired formula for $\nu=k+1$ and with condition (4). To obtain the general case $\nu>k+1$, we pursue the integrations by parts.

It remains to estimate the various terms of (3). We choose $\nu>(n / 2)+$ $2 k+(1 / 2)$ and we recall the classical properties of Bessel functions :

$$
J_{\mu}(t)=O\left(t^{-1 / 2}\right) \quad(t \rightarrow+\infty), \quad J_{\mu}(t)=O\left(t^{\mu}\right) \quad(t \rightarrow 0) .
$$

In the formula (3), we first consider the terms such that $j=0$. From the formula (2), we deduce the following estimations :

$$
h_{m}(t)=O\left(|\log t|^{\alpha-m}(\log |\log t|)^{-\sigma}\right) \quad(t \rightarrow 0),
$$

where $\sigma$ can be replaced by $\sigma+1$ in case $\alpha=0$. By using the conditions

$$
n+2 k-1>-1 \quad, \quad \frac{n}{2}+2 k-\frac{1}{2}-\nu<-1,
$$

and the estimations (6), we obtain

$$
\begin{aligned}
\left|\int_{0}^{\infty} t^{\frac{n}{2}+2 k-\nu} J_{\frac{n}{2}+\nu-1}(r t) \rho(t) h_{m}(t) d t\right| & \\
\leq & c_{1}\left(r^{\frac{n}{2}+\nu-1} \int_{0}^{1 / r} t^{n+2 k-1}|\log t|^{\alpha-m}(\log |\log t|)^{-\sigma} d t\right. \\
& \left.\quad+r^{-1 / 2} \int_{1 / r}^{1 / e^{3}} t^{\frac{n}{2}+2 k-\frac{1}{2}-\nu}|\log t|^{\alpha-m}(\log |\log t|)^{-\sigma} d t\right) \\
\leq & c_{2} r^{\nu-\frac{n}{2}-1-2 k}(\log r)^{\alpha-m}(\log (\log r))^{-\sigma},
\end{aligned}
$$

for $r$ sufficiently large. If we turn to the terms such that $j>0$, we have the following trivial estimation : 


$$
\begin{aligned}
& \left|\int_{0}^{\infty} t^{\frac{n}{2}+2 k-m-l} J_{\frac{n}{2}+\nu-1}(r t) \rho^{(j)}(t) h_{m}(t) d t\right| \\
& \quad \leq c_{1} r^{-1 / 2} \int_{1 / e^{3}}^{1 / e^{2}} t^{\frac{n}{2}+2 k-m-l-\frac{1}{2}}\left|h_{m}(t)\right| d t \leq c_{2} r^{-1 / 2} .
\end{aligned}
$$

That ends up the Proof of Proposition 1.

We give now sharp conditions on the couple $(\alpha, \sigma)$ such that the fonction defined by (1) belongs to the critical Besov spaces. For the sake of completeness, we discuss the same question in the critical Lizorkin-Triebel spaces $F_{p, q}^{n / p}$ (cf. [9] for their definition). Let us define $U_{q}$ as the set of $(\alpha, \sigma) \in \mathbb{R}^{2}$ such that :

- $\alpha=1-\frac{1}{q}$ and $\sigma>1 / q$, or $\alpha<1-\frac{1}{q}$, in case $1<q<\infty$,

- $\alpha=0$ and $\sigma>0$, or $\alpha<0$, in case $q=1$,

- $\alpha=1$ and $\sigma \geq 0$, or $\alpha<1$, in case $q=\infty$.

Proposition 2. Let $f$ be the function defined by (1).

1. If $p, q \in[1,+\infty]$, then $f \in B_{p, q}^{n / p}\left(\mathbb{R}^{n}\right)$ if and only if $(\alpha, \sigma) \in U_{q}$.

2. If $p \in] 1,+\infty\left[\right.$ and $q \in[1,+\infty]$, then $f \in F_{p, q}^{n / p}\left(\mathbb{R}^{n}\right)$ if and only if $(\alpha, \sigma) \in U_{p}$.

Proof. To each couple $(\alpha, \sigma)$, we associate the sequence $\left(\varepsilon_{j}\right)_{j \geq 2}$ defined by

$$
\varepsilon_{j}:=j^{\alpha-1}(\log j)^{-\sigma} \quad \text { if } \quad \alpha \neq 0, \quad \varepsilon_{j}:=j^{-1}(\log j)^{-\sigma-1} \quad \text { if } \quad \alpha=0,
$$

which belongs to $l^{q}$ if and only if $(\alpha, \sigma) \in U_{q}$.

Step 1. Let us assume $(\alpha, \sigma) \in U_{q}$. We are going to prove that $f \in B_{1, q}^{n}\left(\mathbb{R}^{n}\right)$, which the smallest relevant Besov space. We use the Littlewood-Paley setting, so we consider a function $\psi \in \mathcal{D}\left(\mathbb{R}^{n}\right)$, with support in the annulus $1 \leq|\xi| \leq 3$, such that

$$
\sum_{j \in \mathbb{Z}} \psi\left(2^{j} \xi\right)=1 \quad(\forall \xi \neq 0),
$$

and we define the operators $L_{j}$ by

$$
\left(L_{j} f\right)^{\curlyvee}(\xi):=\psi\left(2^{-j} \xi\right) \widehat{f}(\xi) .
$$


Since $f$ is clearly integrable, it suffices to prove the following :

$$
\left(\sum_{j \geq 2}\left(2^{j n}\left\|L_{j} f\right\|_{1}\right)^{q}\right)^{1 / q}<\infty .
$$

To do so, we consider the functions $g_{j}$ defined by $\widehat{g_{j}}(\xi):=\psi(\xi) \widehat{f}\left(2^{j} \xi\right)$. By Proposition 1, we have

$$
\left|\Delta^{k}\left(\widehat{g_{j}}\right)(\xi)\right| \leq c_{k} 2^{-j n} \varepsilon_{j}, \quad \forall \xi \in \mathbb{R}^{n} .
$$

Since $\widehat{g_{j}}$ is the supported by the annulus $1 \leq|\xi| \leq 3$, the estimation (8) implies

$$
|x|^{2 k}\left|g_{j}(x)\right| \leq c_{k}^{\prime} 2^{-j n} \varepsilon_{j}, \quad \forall x \in \mathbb{R}^{n} .
$$

By taking $k>n / 2$ and $k=0$ in (9), we obtain $\left\|g_{j}\right\|_{1} \leq c 2^{-j n} \varepsilon_{j}$. Since $L_{j} f(x)=2^{j n} g_{j}\left(2^{j} x\right)$, we obtain $(7)$.

Step 2. Let us assume $(\alpha, \sigma) \notin U_{q}$. We are going to prove that $f \notin$ $B_{\infty, q}^{0}\left(\mathbb{R}^{n}\right)$, which the biggest relevant Besov space. Let us consider a positive function $\varphi \in \mathcal{D}(] 0,+\infty[)$, such that $\varphi(t)=1$ for $1 \leq t \leq 2$, and define

$$
\theta(x):=|x|^{1-n} \varphi^{\prime}(|x|), \quad \forall x \in \mathbb{R}^{n} .
$$

Then $\theta \in \mathcal{D}\left(\mathbb{R}^{n}\right)$ and $\int \theta(x) d x=0$. If $f$ would belong to $B_{\infty, q}^{0}\left(\mathbb{R}^{n}\right)$, then, by a theorem of Peetre [5, Theorem 4, p.164] (see also [2, Proposition 19]), the sequence defined by

$$
f_{j}(x):=2^{n j} \int \theta\left(2^{j}(x-y)\right) f(y) d y, \quad \forall j \in \mathbb{N},
$$

would satisfy the following property :

$$
\left(\sum_{j \geq 0}\left\|f_{j}\right\|_{\infty}^{q}\right)^{1 / q}<\infty .
$$

Now we are going to prove that

$$
\left(\sum_{j \geq 0}\left|f_{j}(0)\right|^{q}\right)^{1 / q}=\infty,
$$


a property which contradicts (10). If $\omega$ denotes the volume of the unit sphere in $\mathbb{R}^{n}$, we have

$$
\omega^{-1} f_{j}(0)=\int_{0}^{\infty} \varphi\left(2^{j} t\right)\left(-h_{0}^{\prime}(t)\right) \rho(t) d t-\int_{0}^{\infty} \varphi\left(2^{j} t\right) h_{0}(t) \rho^{\prime}(t) d t .
$$

The second above term plays no role, since it is a $O\left(2^{-j}\right)$. On the other hand, by formula (2) we have

$$
\int_{0}^{\infty} \varphi\left(2^{j} t\right)\left(-h_{0}^{\prime}(t)\right) \rho(t) d t \geq c_{1} \int_{2^{-j}}^{2^{-j+1}}\left|h_{1}(t)\right| \frac{d t}{t} \geq c_{2} \varepsilon_{j},
$$

for sufficiently large $j$ 's .

That ends up the proof of (11) and of Proposition 2.

Step 3. According to Jawerth [3] (see also [6, Theorem 2.2.3, p. 31]), the following embeddings hold :

$$
B_{1, p}^{n}\left(\mathbb{R}^{n}\right) \hookrightarrow F_{p, q}^{n / p}\left(\mathbb{R}^{n}\right) \hookrightarrow B_{\infty, p}^{0}\left(\mathbb{R}^{n}\right) .
$$

Then assertion concerning Lizorkin-Triebel spaces is a consequence of the two preceding steps.

Remark. The case of $B_{p, p}^{n / p}\left(\mathbb{R}^{n}\right), 1<p<+\infty$, in Proposition 2, was previously obtained by Triebel [8].

Proposition 3. Let $\beta>0,0<\alpha+\frac{n}{p}<2(\beta+1)$. Let us define

$$
\sigma:=\frac{1}{\beta+1}\left(\alpha+\frac{n}{p}\right) .
$$

If $g \in B_{\infty, \infty}^{\gamma}(\mathbb{R})$ for some $\gamma>\sigma$, then the function

$$
f(x):=|x|^{\alpha} g\left(|x|^{-\beta}\right) \rho(|x|)
$$

belongs to $B_{p, \infty}^{\sigma}\left(\mathbb{R}^{n}\right)$. If moreover $g(t)=\sin ^{2} \frac{t}{2}$, then $f$ does not belong to $B_{p, q}^{\sigma}\left(\mathbb{R}^{n}\right)$ for $q<\infty$.

Proof. Step 1. Case $\sigma<1$. Of course we may assume that $\gamma<1$. Thanks to known properties of power functions, we are reduced to estimate

$$
I(h):=\int_{|x| \leq 1}|x|^{\alpha p}\left|g\left(|x+h|^{-\beta}\right)-g\left(|x|^{-\beta}\right)\right|^{p} d x,
$$


when $h \rightarrow 0$. From the inequality

$$
|| x+\left.h\right|^{-\beta}-\left.|x|^{-\beta}|\leq c| h|| x\right|^{-\beta-1}, \quad \text { for } \quad|h| \leq \frac{|x|}{2},
$$

we deduce

$$
I(h) \leq c\left(|h|^{\gamma p} \int_{|h|^{\frac{1}{\beta+1}} \leq|x| \leq 1}|x|^{\alpha p-\gamma p(\beta+1)} d x+\int_{|x|<|h|^{\frac{1}{\beta+1}}}|x|^{\alpha p} d x\right) .
$$

By assumptions, we have $\alpha p-\gamma p(\beta+1)<-n$ and $\alpha p>-n$. Hence we have

$$
I(h) \leq c|h|^{\frac{\alpha p+n}{\beta+1}},
$$

the wished estimation.

Step 2. Case $\sigma \geq 1$. Now we have to estimate

$$
J(h):=\int_{|x| \leq 1}|x|^{\alpha p}\left|g\left(|x+h|^{-\beta}\right)+g\left(|x-h|^{-\beta}\right)-2 g\left(|x|^{-\beta}\right)\right|^{p} d x .
$$

We shall use the following identity :

$$
\begin{aligned}
2(g & \left.\left(|x+h|^{-\beta}\right)+g\left(|x-h|^{-\beta}\right)-2 g\left(|x|^{-\beta}\right)\right) \\
= & g\left(|x+h|^{-\beta}\right)-g\left(2|x|^{-\beta}-|x-h|^{-\beta}\right)+g\left(|x-h|^{-\beta}\right) \\
& \quad-g\left(2|x|^{-\beta}-|x+h|^{-\beta}\right)+g\left(|x+h|^{-\beta}\right)+g\left(2|x|^{-\beta}-|x+h|^{-\beta}\right) \\
& \quad-2 g\left(|x|^{-\beta}\right)+g\left(|x-h|^{-\beta}\right)+g\left(2|x|^{-\beta}-|x-h|^{-\beta}\right)-2 g\left(|x|^{-\beta}\right) .
\end{aligned}
$$

By combining this identity with (13) and with

$$
|| x+\left.h\right|^{-\beta}+|x-h|^{-\beta}-\left.2|x|^{-\beta}|\leq c| h\right|^{2}|x|^{-\beta-2}, \text { for }|h| \leq \frac{|x|}{2},
$$

we see that $J(h)$ is estimated by

$$
\begin{aligned}
& |h|^{2 p} \int_{|h|^{\frac{1}{\beta+1}} \leq|x| \leq 1}|x|^{\alpha p-p(\beta+2)} d x \\
& \quad+|h|^{\gamma p} \int_{|h|^{\frac{1}{\beta+1}} \leq|x| \leq 1}|x|^{\alpha p-\gamma p(\beta+1)} d x+\int_{|x|<|h|^{\frac{1}{\beta+1}}}|x|^{\alpha p} d x .
\end{aligned}
$$

As in Step 1, the two above last terms are estimated by $|h|^{\frac{\alpha p+n}{\beta+1}}$. Moreover a short discussion shows that

$$
|h|^{2 p} \int_{|h|^{\frac{1}{\beta+1}} \leq|x| \leq 1}|x|^{\alpha p-p(\beta+2)} d x=O\left(|h|^{r p}\right)
$$


for some $r>\sigma$.

Step 3. Now we prove the estimation from below in case of $g(t)=\sin ^{2} \frac{t}{2}$.

We give the proof for $\sigma \geq 1$ (the case $\sigma<1$ is similar and easier). Assume

$|h| \leq c_{1}|x|^{\beta+1}$, with $c_{1}$ sufficiently small. Then by (13) we have

$$
\begin{aligned}
& \mid g\left(|x+h|^{-\beta}\right)+g\left(2|x|^{-\beta}-|x+h|^{-\beta}\right)-2 g\left(|x|^{-\beta}\right) \\
& \quad+g\left(|x-h|^{-\beta}\right)+g\left(2|x|^{-\beta}-|x-h|^{-\beta}\right)-2 g\left(|x|^{-\beta}\right) \mid \\
& \quad=\left.|\cos | x\right|^{-\beta}|| \cos \left(|x+h|^{-\beta}-|x|^{-\beta}\right)+\cos \left(|x-h|^{-\beta}-|x|^{-\beta}\right)-2 \mid \\
& \quad \geq\left.\left. c_{2}|\cos | x\right|^{-\beta}|| h\right|^{2}|x|^{-2(\beta+1)} .
\end{aligned}
$$

By using again the identity, we see that $J(h)$ is greater than

$$
\begin{aligned}
& c_{3}|h|^{2 p} \int_{c_{2}|h|^{\frac{1}{\beta+1}} \leq|x| \leq 1}|x|^{\alpha p-2 p(\beta+1)}\left|\cos \left(|x|^{-\beta}\right)\right|^{p} d x-c_{4}|h|^{2 p} \\
& \quad \times \int_{c_{2}|h|^{\frac{1}{\beta+1}} \leq|x| \leq 1}|x|^{\alpha p-p(\beta+2)} d x .
\end{aligned}
$$

It is easily seen that

$$
\int_{\varepsilon \leq|x| \leq 1}|x|^{a}\left|\cos \left(|x|^{-\beta}\right)\right|^{p} d x \approx \varepsilon^{a+n} \quad(\varepsilon \rightarrow 0),
$$

for any $a<-n$. Then $J(h) \geq c_{5}|h|^{\frac{\alpha p+n}{\beta+1}}$, for $|h|$ sufficiently small.

\section{Proofs of the main results}

3.1 Proof of Theorem 1. This theorem is a classical result. We outline the proof, for the sake of completeness. The function $t \mapsto \rho(|t|)^{\mu}|t|^{\mu}$ does not belong to $B_{p, q}^{\mu+(1 / p)}(\mathbb{R})$, for $q<+\infty$ (cf. for instance $[6,2.3 .1$, p. 44]). Hence the function defined by $f(x):=x_{1} \rho(|x|)$, for $x \in \mathbb{R}^{n}$, belongs to $\mathcal{D}\left(\mathbb{R}^{n}\right)$, but $|f|^{\mu}$ does not belong to $B_{p, q}^{\mu+(1 / p)}\left(\mathbb{R}^{n}\right)$ for $q<+\infty$.

3.2 Proof of Theorem 2. Step 1. Assume that $s<n / p$. Let us take $\alpha$ such that

$$
\frac{1}{\mu}\left(\frac{n}{p}-s\right)<\alpha<\frac{n}{p}-s .
$$

According to $\left[6,2.3 .1\right.$, p. 44], the searched function is $f(x):=|x|^{-\alpha} \rho(|x|)$.

Step 2. Assume that $s=n / p$ and $q>1$. We consider the function $f$ defined by (1), with $\alpha=1-\frac{1}{q}, \sigma q>1$. According to Proposition 2, one has $f \in B_{p, q}^{n / p}\left(\mathbb{R}^{n}\right)$ and $f^{\mu} \notin B_{p, q}^{n / p}\left(\mathbb{R}^{n}\right)$. 
3.3 Proof of Theorem 3. Following a remark of Sickel [7, Par. 3.4], we can assume that $n=1$. Indeed tensorizing with a $C^{\infty}$ compactly supported function of the remaining variables gives the general case.

Step 1. For $s>1 / p$, we use the same test functions as in the proof of Theorem 2. We take $\alpha$ such that

$$
s-\frac{1}{p}<\alpha<\frac{1}{\mu}\left(s-\frac{1}{p}\right)
$$

then we set $f(x):=|x|^{\alpha} \rho(|x|)$. We obtain $f \in B_{p, q}^{s}(\mathbb{R})$ whereas $f^{\mu} \notin$ $B_{p, q}^{s}(\mathbb{R})$.

Step 2 . Now we assume $s \leq 1 / p$. Then the relevant power functions are not bounded. Instead we use the bounded functions with great oscillations at 0 constructed in Proposition 3. Let us take

$$
\beta>\max \left(0, \frac{1}{s p}-1\right), \quad s(\beta+1)-\frac{1}{p}<\alpha<\frac{1}{\mu}\left(s(\beta+1)-\frac{1}{p}\right) .
$$

Let us consider the function $f$ defined by (12), with $n=1$ and

$$
g(t):=\left(\sin ^{2} \frac{t}{2}\right)^{1 / \mu}
$$

Since $g \in B_{\infty, \infty}^{1 / \mu}(\mathbb{R})$, we obtain $f \in B_{p, q}^{s}(\mathbb{R})$. Since we have

$$
f(x)^{\mu}=|x|^{\alpha \mu} \sin ^{2}\left(\frac{1}{2}|x|^{-\beta}\right)
$$

in a neighborhood of 0 , the second part of Proposition 3 yields $f^{\mu} \notin B_{p, q}^{s}(\mathbb{R})$.

Acknowledgments. We thank one of the referees, who indicated us the extension of Proposition 2 to Lizorkin-Triebel spaces and Professor Triebel for useful discussions. 


\section{References}

[1] G. Bourdaud, Fonctions qui opèrent sur les espaces de Besov et de Triebel, Annal. I.H.P. - Anal. non linéaire, 10 (1993), 413-422.

[2] G. Bourdaud, Ce qu'il faut savoir sur les espaces de Besov, Prépub. Univ. Paris 7 - URA 212, 53 (janvier 1993).

[3] B. Jawerth, Some observations on Besov and Triebel-Lizorkin spaces, Math. Scand. 40, (1977), 94-104.

[4] D. Kateb, On the boundedness of the mapping $f \mapsto|f|^{\mu}, \mu>1$, on Besov spaces, Math. Nachr., 248-249 (2003), 110-128.

[5] J. Peetre, New thoughts on Besov spaces, Duke Univ. Math. Series I, (1976).

[6] T. Runst and W. Sickel, Sobolev Spaces of Fractional Order, Nemytskij Operators, and Nonlinear Partial Differential Equations, De Gruyter, 1996.

[7] W. Sickel, Boundedness Properties of the Mapping $f \mapsto|f|^{\mu}, 0<\mu<1$ in the Framework of Besov Spaces, Preprint, FSU Jena, (1998).

[8] H. Triebel, Approximation numbers and entropy numbers of embeddings of fractional Besov-Sobolev spaces in Orlicz spaces, Proc. London Math. Soc, 66 (1993), 589-618.

[9] H. Triebel, Theory of Function Spaces II, Birkhäuser, 1992.

[10] S. Wainger, Special Trigonometric Series in $k$ Dimensions, Mem. Amer. Math. Soc., 59 (1965).

[11] G.N. Watson, Theory of Bessel Functions, Cambridge University Press, 1944.

Institut de Mathématiques de Jussieu

Équipe d'Analyse Fonctionnelle

Case 186

4 place Jussieu, 75252 Paris Cedex 05

France

(E-mail : bourdaud@ccr.jussieu.fr)

(Received : March 2003) 


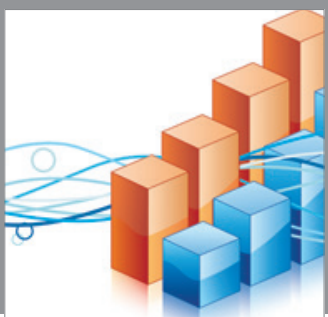

Advances in

Operations Research

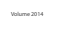

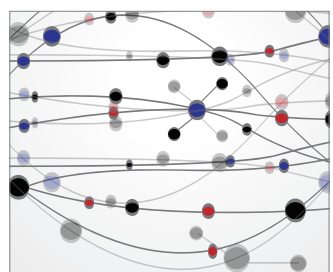

\section{The Scientific} World Journal
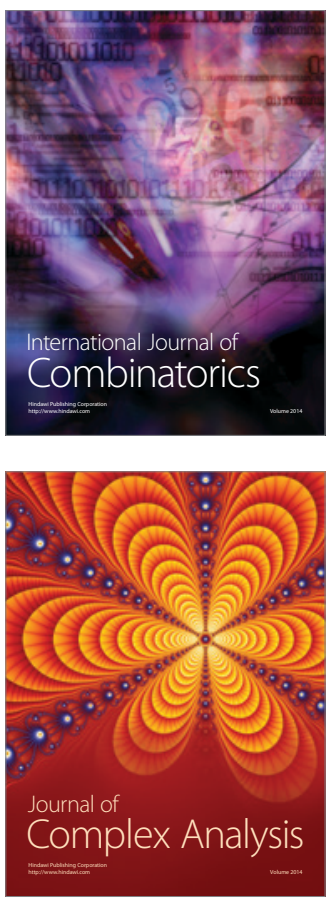

International Journal of

Mathematics and

Mathematical

Sciences
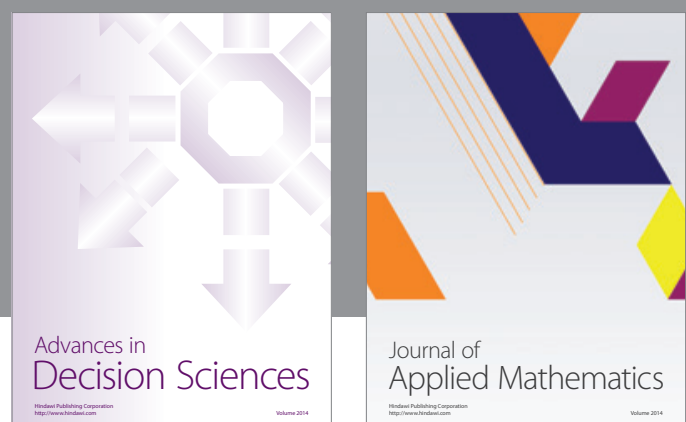

Journal of

Applied Mathematics
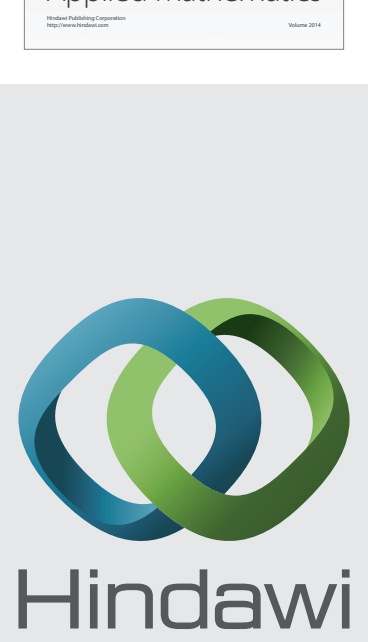

Submit your manuscripts at http://www.hindawi.com
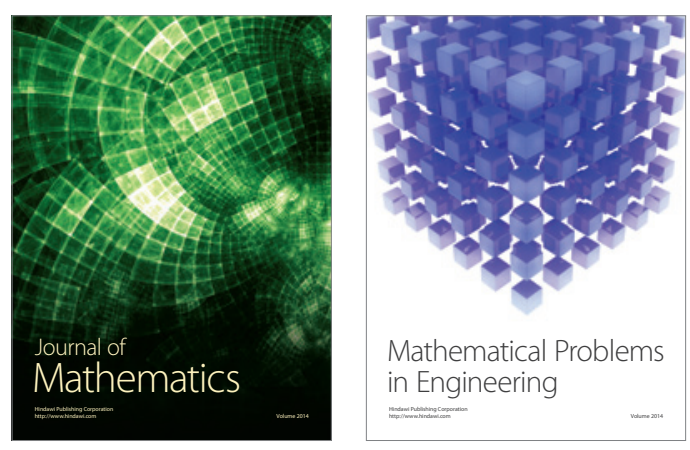

Mathematical Problems in Engineering
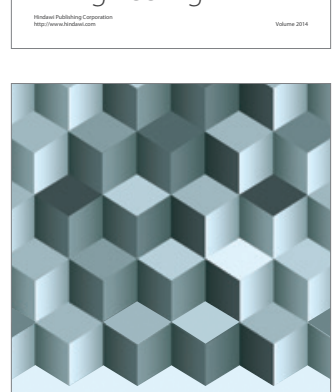

Journal of

Function Spaces
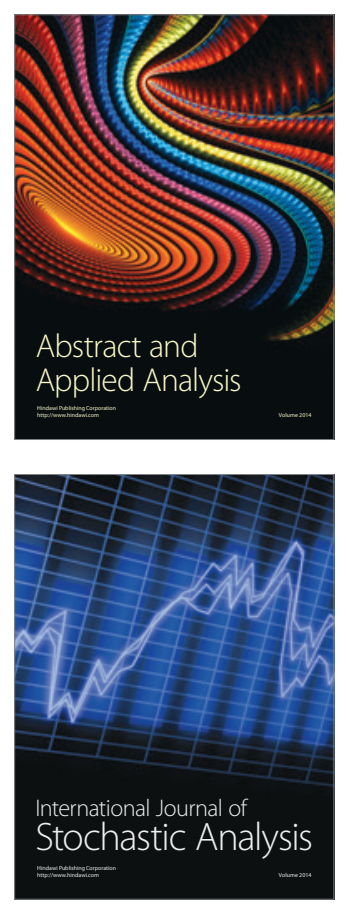

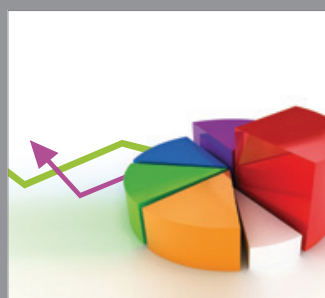

ournal of

Probability and Statistics

Promensencen
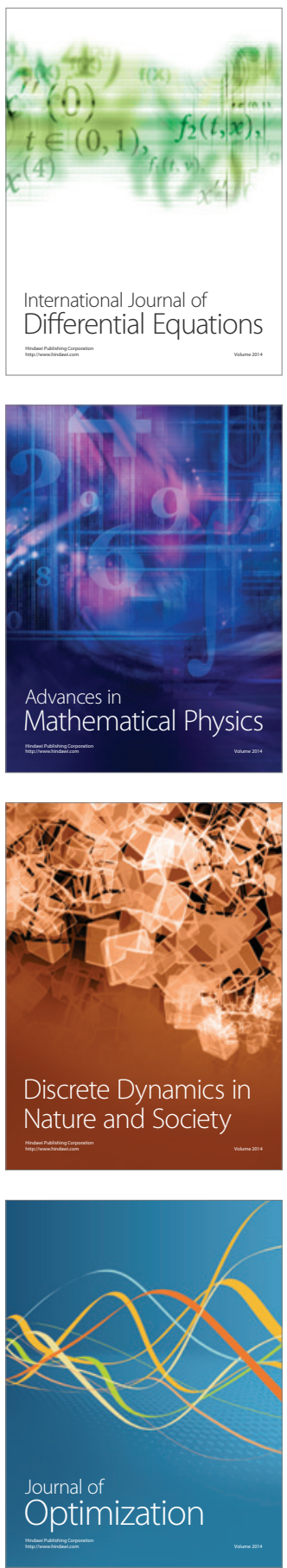